\title{
Tratamento com Radio e Quimioterapia do Carcinoma Epidermóide do Canal Anal: Experiência do Hospital Barão de Lucena
}

\author{
Radiochemotherapy for Squamous Cell Carcinoma of the Anal Canal: \\ Barao de Lucena Hospital Experience
}

\author{
MAURILIOTOSCANO DE LUCENA ${ }^{2}$, ADAUTO BARROS ${ }^{1}$, ANTÔNIO BOOZ ${ }^{2}$, CARLOS LOYO ${ }^{2}$, CARLOS UCHÔA $^{3}$, \\ ERNESTO ROESLER ${ }^{6}$, FERNANDO MONTEIRO $^{1}$, FRANCISCO SARMENTO ${ }^{2}$, JOAQUIM HERBÊNIO ${ }^{2}$, JOSÉEDSON DOS \\ SANTOS $^{2}$, MARCELO PADILHA ${ }^{5}$, MAURÍCIO MATOS ${ }^{2}$, PAULA GOMES LOBO ${ }^{4}$, ROBERTO REGIS ${ }^{1}$, ROBERTA \\ TORREÃO ${ }^{5}$, ROMILDOARAÚJO $^{5}$, ROGÉRIOLEAL $^{2}$
}

${ }^{I}$ Coloproctologista do Hospital Barão de Lucena, ${ }^{2}$ Coloproctologista do Hospital Barão de Lucena e Titular da Sociedade Brasileira de Coloproctologia, ${ }^{3}$ Coloproctologista do Hospital Barão de Lucena e Associado da Sociedade Brasileira de Coloproctologia, ${ }^{4}$ Coloproctologista do Hospital Barão de Lucena e Filiado da Sociedade Brasileira de Coloproctologia, ${ }^{5}$ Oncologista do Hospital Barão de Lucena, ${ }^{6}$ Radioterapeuta do Instituto Ivo Roesler.

\begin{abstract}
LUCENA MT; BARROS A; BOOZ A; LOYO C; UCHÔA C; ROESLER E; MONTEIRO F; SARMENTO F; HERBÊNIO J; SANTOS JE; PADILHA M; MATOS M; LOBO PG; REGIS R; TORREÃO R; ARAÚJO R; LEAL R. Tratamento com Radio e Quimioterapia do Carcinoma Epidermóide do Canal Anal: Experiência do Hospital Barão de Lucena. Rev bras Coloproct, 2010;30(2): 167-174.

RESUMO: Objetivos: Apresentar os resultados e analisar as variáveis implicadas no tratamento e prognóstico do carcinoma epidermóide do canal anal tratado através da radio e quimioterapia no Hospital Barão de Lucena-SUS-PE. Metodologia: Análise dos prontuários de pacientes com diagnóstico de câncer do canal anal submetidos a tratamento radioquimioterápico. O período de acompanhamento foi de junho de 1989 a junho de 2005. Foram incluídos os pacientes com diagnóstico histológico de câncer de canal anal, enquadrados nos estadios I, II, IIIa e IIIb, submetidos a dois ciclos de quimioterapia com 5-fluorouracil (5-FU) na dose de $1 \mathrm{~g} / \mathrm{m}^{2} /$ dia em infusão contínua de 96 horas e cisplatino na dose de $100 \mathrm{mg} / \mathrm{m}^{2}$ administrado em 6 horas no segundo dia de infusão de cada ciclo, administrados na primeira e terceira semanas do esquema de tratamento radioterápico. Resultados: Avaliamos 108 prontuários de pacientes que preencheram os critérios do protocolo. $O$ tempo médio de seguimento foi de 51 meses (1-182 meses). Houve predomínio do gênero feminino ( $81,5 \%$ dos pacientes). A idade variou de 33 a 83 anos (média de 59 anos). $O$ tipo histológico mais freqüente foi o carcinoma de células escamosas (80,6\% dos casos). Em 21 pacientes, foi diagnosticado carcinoma basalóide. Quanto ao grau de diferenciação, prevaleceu o tipo moderadamente diferenciado (61\% dos pacientes com carcinoma de células escamosas). $O$ índice de resposta inicial completa foi de $\mathbf{8 9 , 8 \%}$. Onze pacientes persistiram com tumor após o tratamento radio e quimioterápico. O índice de resposta inicial completa foi menor nos estadios IIIa e IIIb em relação aos estadios I e II com significância estatística $(\mathbf{p}<0,05) .14$ pacientes evoluíram com recidiva tumoral, oito com recidiva local $(7,4 \%)$ e seis $(5,5 \%)$ com recidiva linfática e à distância. CONCLUSÕES: O tratamento radioquimioterápico exclusivo do carcinoma epidermóide do canal anal, tem índice de resposta completo bastante elevado com morbidade aceitável. $O$ tratamento cirúrgico ainda tem seu valor nos casos de persistência da lesão e/ou de recidiva local, com resultados satisfatórios.
\end{abstract}

Descritores: Neoplasia do ânus, neoplasia de células escamosas, radioterapia, quimioterapia, quimioterapia combinada.

\section{INTRODUÇÃO}

O câncer do canal anal é infreqüente e corresponde a cerca de $2 \%$ das neoplasias malignas do intestino grosso. Ocorre com mais freqüên- cia no sexo feminino numa proporção de $2: 1$ e acomete principalmente pacientes na sexta e sétima décadas de vida. Com relação ao tipo histológico, cerca de $80 \%$ destes tumores são carcinomas espinocelulares ${ }^{1,8}$.

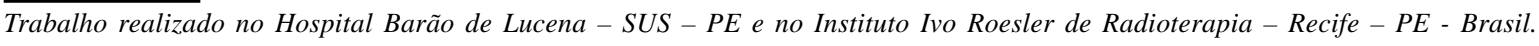

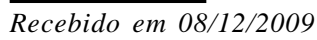

Aceito para publicação em 07/01/2010 
Tratamento com Radio e Quimioterapia do Carcinoma Epidermóide do Canal Anal: Experiência do Hospital Barão de Lucena

Maurilio Toscano de Lucena e Cols.
Vol. 30
Sua incidência aumentou após a década de 80 , provavelmente em função da transmissão sexual do papiloma vírus humano ${ }^{1}$. Vários outros fatores têm sido associados com o câncer do canal anal como: feridas anais crônicas ou irritação crônica da pele perianal, tabagismo, infecções virais e bacterianas, imunossupressão e exposição prévia à radiação².

É importante a distinção anatômica entre o canal anal e a margem anal, pois a história natural do câncer nessas regiões é diferente. A "American Joint Committee on Cancer" (AJCC) e a "Union Internationale Contra le Cancer" (UICC) estabeleceram que o canal anal estende-se do anel anorretal (2 $\mathrm{cm}$ acima da linha pectínea) à borda anal, o que é aceito pelo atual sistema de estadiamento ${ }^{3}$. A drenagem linfática do canal anal depende da localização do tumor: acima da linha pectínea é feita para os linfonodos perirretais e paravertebrais, assim como no adenocarcinoma do reto. Abaixo desta, drena para os gânglios inguinais e femorais.

O estadiamento deste câncer, obedece aos critérios estabelecidos pela AJCC/ UICC, que o divide segundo as categorias $\mathrm{T}$ (extensão do tumor primário), $\mathrm{N}$ (ausência ou presença e extensão de metástases linfonodais regionais) e $M$ (presença ou ausência de metástases à distância) ${ }^{3}$. Os tumores menores que dois centímetros e os bem diferenciados têm melhor prognóstico ${ }^{4}$.

No que se refere ao tratamento, a cirurgia de ressecção abdômino-perineal com colostomia definiti- va era o tratamento eleito, porém desde a publicação de Nigro et al em 1974, a combinação de rádio e quimioterapia tornou-se o tratamento de escolha para o carcinoma epidermóide do canal anal. No entanto, em pacientes com persistência da doença ou recidiva local, um tratamento cirúrgico de resgate deve ser instituído $8,14,15$.

O tratamento conservador de esfíncter anal através da radioterapia associada à quimioterapia utilizando o 5-Fluorouracil + Cisplatino vem sendo utilizado como terapia de escolha para o carcinoma epidermóide do canal anal no Hospital Barão de LucenaSUS-PE desde 1989. O objetivo do presente estudo, foi avaliar os resultados e analisar as variáveis implicadas no tratamento e prognóstico desta lesão.

\section{MÉTODOS}

O estudo foi realizado através da análise dos prontuários de pacientes com diagnóstico de câncer do canal anal atendidos no ambulatório de coloproctologia do Hospital Barão de Lucena e submetidos a tratamento radioquimioterápico segundo protocolo do serviço.

O período de acompanhamento foi de junho de 1989 a junho de 2005.

Foram incluídos os pacientes com diagnóstico confirmado de câncer de canal anal, através de biópsia da lesão e exame histopatológico, enquadrados nos estadios I, II, IIIa e IIIb (tabela 1), tratados com radioquimioterapia segundo protocolo.

Tabela 1 - Estadiamento TNM para Câncer do Canal Anal (AJCC/UICC).

\begin{tabular}{|c|c|c|c|}
\hline T - tumor primário & $\mathrm{N}$ - linfonodos & M - Metástases & Estagio $(\mathbf{E})$ \\
\hline T0 - sem evidência de tumor & $\begin{array}{l}\text { N0 - sem metastases para } \\
\text { linfonodos regionais }\end{array}$ & $\begin{array}{l}\text { M0 - sem metástases } \\
\text { à distância }\end{array}$ & $0-$ TisNOM0 \\
\hline Tis - carcinoma in situ & & & I - T1NOM0 \\
\hline $\mathrm{T} 1-\leq 2 \mathrm{~cm}$ & $\mathrm{~N} 1$ - linfonodos perirretais & $\begin{array}{l}\text { M1 - metástases } \\
\text { à distância }\end{array}$ & II - T2,3N0M0 \\
\hline $\mathrm{T} 2->2 \mathrm{e}<5 \mathrm{~cm}$ & $\begin{array}{l}\mathrm{N} 2 \text { - linfonodos unilaterais } \\
\text { (cadeia iliaca interna ou inguinal) }\end{array}$ & & $\begin{array}{c}\text { III a - T1-3N1M0 } \\
\text { T4N0M0 }\end{array}$ \\
\hline $\mathrm{T} 3->5 \mathrm{~cm}$ & $\begin{array}{l}\mathrm{N} 3-\mathrm{N} 1 \text { ou } \mathrm{N} 2 \text { e/ou cadeia } \\
\text { iliaca interna ou inguinal bilateral }\end{array}$ & & $\begin{array}{l}\text { III b - T4N1M0 } \\
\text { Qualquer TN2M0 } \\
\text { Qualquer TN3M0 }\end{array}$ \\
\hline $\begin{array}{l}\mathrm{T} 4 \text { - qualquer tamanho com } \\
\text { invasão de órgãos adjacentes } \\
\text { (vagina, uretra, bexiga) }\end{array}$ & & & $\begin{array}{l}\text { IV - Qualquer T } \\
\text { Qualquer N M1 }\end{array}$ \\
\hline
\end{tabular}


Pacientes que não completaram o tratamento, ou não fizeram uso das duas drogas da quimioterapia, ou que tiveram seu tratamento suspenso por um período maior que 30 dias, ou os que já haviam sido submetidos a tratamento radioterápico previamente, foram excluídos do estudo.

$\mathrm{O}$ estadiamento foi feito conforme os critérios estabelecidos pela AJCC/UICC, descrito anteriormente.

Os pacientes foram acompanhados ambulatorialmente através do exame clínico e exames complementares a cada 2 meses nos dois primeiros anos, com intervalo de 6 meses até o quinto ano e então anualmente. Foi considerada resposta inicial completa quando, cerca de 4 a 6 semanas após o término do tratamento radioquimioterápico, o paciente tenha apresentado regressão completa da lesão, observada clinicamente ou através de biópsia. A recidiva foi definida como a identificação de lesão tumoral, clinicamente ou através de biópsia, a qualquer tempo após o paciente ter sido considerado como tendo resposta inicial completa.

Os efeitos tóxicos da radioquimioterapia foram avaliados através de escalas de morbidades agudas e tardias elaboradas pelo "Radiation Therapy Oncology Group" (RTOG), pela "European Organization for Reserch and Treatment of Cancer" (EORTC), e pela escala da "Eastern Cooperative Oncology Group" (ECOG), conforme demonstrado na tabela 2.

O tratamento radioterápico foi sempre efetuado em equipamentos de megavoltagem e efetuado através de campos paralelos opostos que englobam a pélvis e as regiões inguinais bilaterais. As regiões inguinais foram sempre tratadas profilaticamente mesmos nos casos N0, com intuito de reduzir o índice de conversibilidade que é de aproximadamente $17 \%$.

Os campos de radiação tiveram seu limite superior entre L5/S1 e o inferior com margem suficiente para englobar todo o períneo. A dose total variou de 4500 - 5040 cGy divididos em sessões diárias de 180 cGy cada.

Os campos foram reduzidos ao se atingir 3060 cGy, para campos menores de acordo com tamanho, extensão e invasão tumoral, conforme o critério de avaliação do médico assistente no momento desta redução. As regiões inguinais, também foram excluídas neste momento, a não ser nos casos de conhecimento de comprometimento inguinal por neoplasia. Neste caso o lado acometido ou ambos continuaram dentro dos campos de irradiação até a conclusão do tratamento.

Em casos de persistência do tumor, detectada através de toque retal ou do exame histopatológico, o paciente recebeu uma dose suplementar de radio-

Tabela 2 - Tabela de Score de Morbidade Aguda do RTOG - ECOG.

\begin{tabular}{|c|c|c|c|c|}
\hline \multicolumn{5}{|c|}{ Score } \\
\hline Órgão/Tecido & Grau 1 & Grau 2 & Grau 3 & Grau 4 \\
\hline Pele & $\begin{array}{l}\text { Eritema discreto ou } \\
\text { folicular, descamação seca, } \\
\text { diminuição da sudorese }\end{array}$ & $\begin{array}{l}\text { Eritema doloroso ou } \\
\text { forte, descamação úmida, } \\
\text { edema moderado }\end{array}$ & $\begin{array}{l}\text { Descamação úmida, } \\
\text { edema importante }\end{array}$ & $\begin{array}{l}\text { Ulceração, hemorragia, } \\
\text { necrose }\end{array}$ \\
\hline GIBaixo & $\begin{array}{l}\text { Alteração dos hábitos } \\
\text { intestinais sem requerer } \\
\text { antidiarréicos, desconforto retal }\end{array}$ & $\begin{array}{l}\text { Diarréia requerendo } \\
\text { medicação, descarga mucosa, } \\
\text { dor retal ou abdominal } \\
\text { requerendo analgésicos }\end{array}$ & $\begin{array}{l}\text { Diarréia requerendo } \\
\text { suporte parenteral, } \\
\text { descarga mucosa ou } \\
\text { sanguínea severa, } \\
\text { distensão abdominal }\end{array}$ & $\begin{array}{l}\text { Obstrução aguda ou } \\
\text { subaguda, fístula ou } \\
\text { perfuração, sangramento } \\
\text { GI que necessite } \\
\text { transfusão sang., dor } \\
\text { abd ou tenesmo neces- } \\
\text { sitando de descompressão. }\end{array}$ \\
\hline Leucócitos & $3.000-4.000$ & $2.000-3.000$ & $1.000-2.000$ & $<1.000$ \\
\hline Plaquetas & $75.000-100.000$ & $50.000-75.000$ & $25.000-50.000$ & $\begin{array}{l}<25.000 \text { ou } \\
\text { sangramento espontâneo }\end{array}$ \\
\hline Hemoglobina & 9,5 & $9,5-7,5$ & $7,5-5,0$ & \\
\hline
\end{tabular}


terapia exclusiva de 1.000 cGy (200 cGy/dia em 5 dias).

Os pacientes foram submetidos a dois ciclos de quimioterapia com 5-fluorouracil (5-FU) na dose de $1 \mathrm{~g} / \mathrm{m}^{2} /$ dia em infusão continua de 96 horas e cisplatino na dose de $100 \mathrm{mg} / \mathrm{m}^{2}$ administrado em 6 horas no segundo dia de infusão de cada ciclo. Os ciclos de quimioterapia foram ministrados na primeira e terceira semanas do esquema de tratamento radioterápico, a partir do primeiro e do vigésimo primeiro dia do tratamento.

Foram analisados os dados com referência à resposta ao tratamento radioquimioterápico avaliando variáveis como estadiamento, tipo histológico, gênero, idade e complicações próprias do tratamento. As variáveis qualitativas foram expressas através de suas proporções e analisadas utilizando-se o teste do qui-quadrado ou o teste Exato de Fisher, quando as frequiências esperadas eram menores que cinco. Foram considerados significantes valores de $p<0,05$. Utilizamos para os cálculos estatísticos, o programa Epi Info versão 3.5.

\section{RESULTADOS}

Foram avaliados 108 prontuários de pacientes que preencheram os critérios do protocolo. $\mathrm{O}$ tempo médio de seguimento foi de 51 meses, variando de 1 a 182 meses. Houve predominância do gênero feminino, que totalizou $88(81,5 \%)$ pacientes. A idade variou de 33 a 83 anos, com média de 59 anos.

O tipo histológico mais freqüente foi o carcinoma de células escamosas, encontrado em 87 (80,6\%) casos. Nos outros 21 pacientes, foi diagnosticado car- cinoma basalóide (figura 1). Quanto ao grau de diferenciação, prevaleceu o tipo moderadamente diferenciado, ocorrendo em $61 \%$ dos pacientes com carcinoma de células escamosas. O tipo basalóide não é classificado de acordo com grau de diferenciação.

$\mathrm{Na}$ época do diagnóstico, $2(1,9 \%)$ pacientes encontravam-se no estadio clínico I, $73(67,6 \%)$ no estádio II, $12(11,1 \%)$ no estadio IIIa e, 21(19,4\%) no estádio IIIb (tabela 3 ).

O índice de resposta inicial completa foi de $89,8 \%$. Onze pacientes persistiram com tumor após tratamento inicial com as doses habituais do protocolo de tratamento. Destes, seis foram submetidos à dose complementar da radioterapia (boost), havendo resposta satisfatória em mais dois pacientes. Com relação aos demais casos, dois encontram-se vivos com tumor, dois foram submetidos à amputação do reto e o último foi a óbito após cirurgia paliativa (colostomia).

As variáveis idade, gênero e tipo histológico do tumor não foram estatisticamente significantes quanto a resposta final ao tratamento.

Tabela 3 - Estadiamento Clínico.

\begin{tabular}{lcc}
\hline & \multicolumn{2}{c}{ Número de Pacientes } \\
\cline { 2 - 3 } Estadiamento & $\mathbf{n}$ & $\mathbf{\%}$ \\
\hline I & 2 & 1,9 \\
II & 73 & 67,6 \\
IIIa & 12 & 11,1 \\
IIIb & 21 & 19,4 \\
Total & 108 & 100 \\
\hline
\end{tabular}

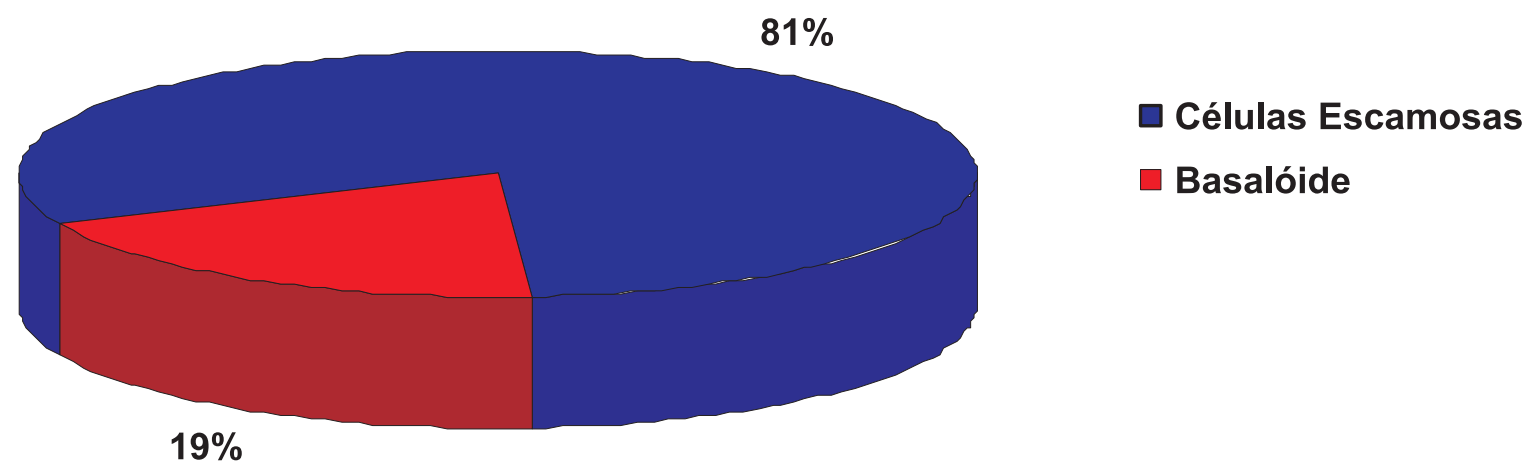

Figura 1 - Frequência do Tipo Histológico. 
Tabela 4 - Correlação do Estadiamento com o Índice de Resposta Inicial Completa.

\begin{tabular}{|c|c|c|c|c|c|c|}
\hline \multirow{3}{*}{ Estadiamento } & \multicolumn{6}{|c|}{ Resposta Inicial Completa } \\
\hline & \multicolumn{2}{|c|}{ Sim } & \multicolumn{2}{|c|}{ Não } & \multicolumn{2}{|c|}{ Total } \\
\hline & $\mathbf{n}$ & $\%$ & $\mathbf{n}$ & $\%$ & $\mathbf{n}$ & $\%$ \\
\hline I / II & 71 & 65,7 & 4 & 3,7 & 75 & 69,4 \\
\hline IIIa / IIIb & 26 & 24 & 7 & 6,6 & 33 & 30,6 \\
\hline Total & 97 & 89,7 & 11 & 10,3 & 108 & 100 \\
\hline
\end{tabular}

* $P=0,03$ (Teste Exato de Fisher).

Agrupando os pacientes com estadiamento I e II e aqueles com IIIa e IIIb, isto é, agrupando os estadiamentos sem e com comprometimento de linfonodos, respectivamente, e correlacionando com o índice de resposta inicial completa, observamos que a resposta foi menor nos estadios IIIa e IIIb do que nos estadios I e II com significância estatística $(p<0,05)$, como mostrado na tabela 4.

Um total de 14 (13\%) pacientes evoluiu com recidiva tumoral, oito com recidiva local $(7,4 \%)$ e seis $(5,5 \%)$ com recidiva em gânglios linfáticos e à distância. O tempo de recidiva médio foi de 19 meses, variando de 3 a 60 meses. Dos pacientes que evoluíram com recidiva local, seis foram submetidos à amputação do reto e destes, quatro encontram-se sem tumor.

Quanto à toxicidade aguda do tratamento radioquimioterápico, foram relatadas algumas complicações com graus variados de gravidade, levando em alguns casos à suspensão temporária do tratamento. Epitelite ocorreu em 49 pacientes, sendo considerada graus 1 e 2 em $39(79,6 \%)$ e graus 3 e 4 em $10(20,4 \%)$ pacientes. Trinta e um pacientes apresentaram diarréia, sendo considerada como de grau 3 em 7 casos, ocasionando a suspensão do tratamento por períodos variáveis. A leucopenia levando ao adiamento do esquema de radioquimioterapia foi observada em três casos. A interrupção temporária do tratamento foi necessária em $24(22,2 \%)$ pacientes, com tempo médio de interrupção de 10 dias, variando de 1 a 30 dias. Com relação às complicações tardias, a mais freqüente foi a estenose de reto, ocorrendo em 11 pacientes. Alguns deles, responderam à realização de dilatação instrumental e outros necessitaram de retotomia ou colostomia derivativa (Tabela 5).

Atualmente, 80 (76,9\%) pacientes encontramse sem evidência de doença, três persistem com doença, e quatro perderam seguimento após resposta inicial completa. A mortalidade nos pacientes estudados foi de $20,2 \%$, sendo que sete $(6,7 \%)$ pacientes faleceram de causas não relacionadas ao tumor (figura 2).

Tabela 5 - Complicações Precoces e Tardias Observadas durante e após tratamento radioquimioterápico.

\begin{tabular}{|c|c|c|c|c|c|c|c|c|c|c|}
\hline \multirow{3}{*}{ Complicações } & \multicolumn{10}{|c|}{ Score de Morbidade Aguda do RTOG - ECOG } \\
\hline & \multicolumn{2}{|c|}{1} & \multicolumn{2}{|c|}{2} & \multicolumn{2}{|c|}{3} & \multicolumn{2}{|c|}{4} & \multicolumn{2}{|c|}{ Total } \\
\hline & $\mathbf{n}$ & $\%$ & $\mathbf{n}$ & $\%$ & $\mathbf{n}$ & $\%$ & $\mathbf{n}$ & $\%$ & $\mathbf{n}$ & $\%$ \\
\hline Epitelite & 8 & 7,4 & 31 & 28,7 & 9 & 8,3 & 1 & 0,9 & 49 & 45,4 \\
\hline Diarréia & 9 & 8,3 & 15 & 13,9 & 7 & 6,5 & & - & 31 & 28,7 \\
\hline Leucopenia & & - & 2 & 1,9 & 1 & 0,9 & & - & 3 & 3,8 \\
\hline Anemia & & - & 1 & 0,9 & & - & & - & 1 & 0,9 \\
\hline Cistite & & - & 1 & 0,9 & & - & & - & 1 & 0,9 \\
\hline Alopecia & & - & 3 & 2,7 & 2 & 1,9 & & - & 5 & 4,6 \\
\hline Retite & 4 & 3,7 & 5 & 4,6 & & - & & - & 9 & 8,3 \\
\hline Estenose de Reto & 4 & 3,7 & 4 & 3,7 & 3 & 2,7 & & - & 11 & 10,1 \\
\hline Fístula Retovaginal & & - & 1 & 0,9 & 1 & 0,9 & & - & 2 & 1,9 \\
\hline
\end{tabular}




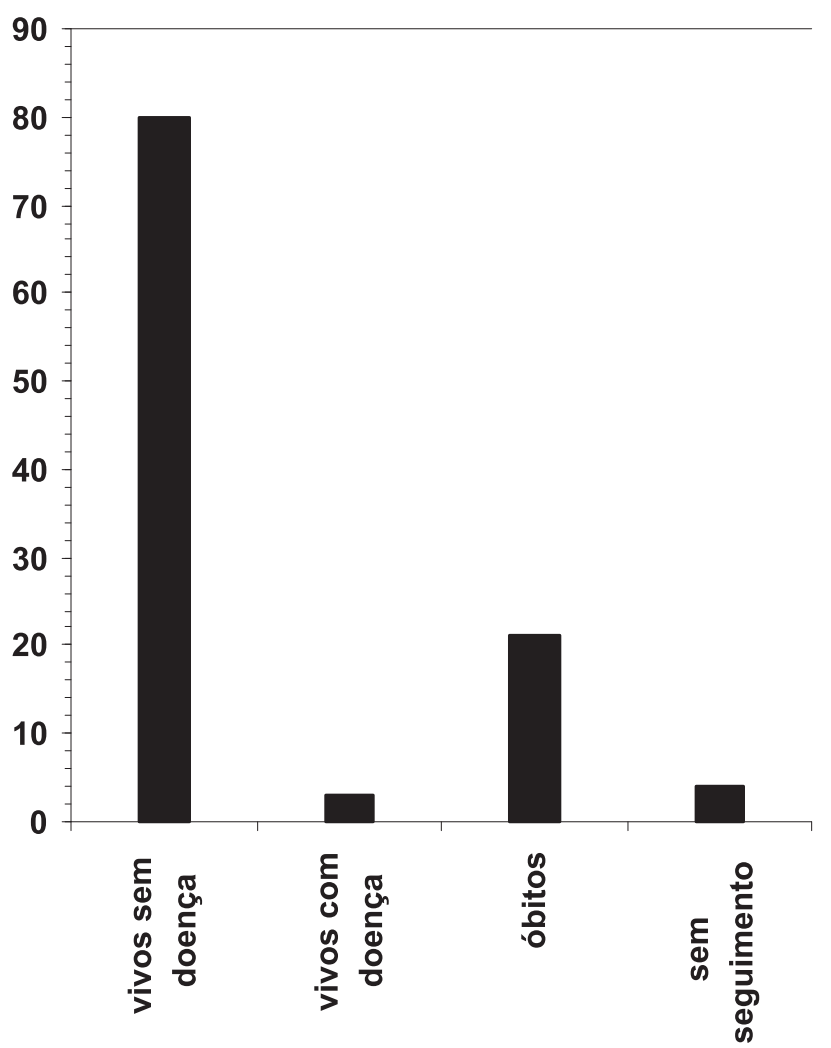

Figura 2 - Situação atual dos pacientes estudados.

\section{DISCUSSÃO}

O tratamento do câncer do canal anal sofreu mudanças significativas a partir da década de 70 , com os relatos de Nigro questionando a necessidade do tratamento cirúrgico com amputação do reto e conseqüente sacrifício do esfíncter anal. Atualmente, já está estabelecida a superioridade do tratamento combinado de radio e quimioterapia para este tipo de câncer, reservando a cirurgia para resgate após falha no tratamento conservador ou nos casos de doença destrutiva do esfíncter anal.

Alguns estudos randomizados como o UKCCCR Anal Cancer Trial Working Party, de 1996, compararam o tratamento combinado com o tratamento pré-operatório radioterápico exclusivo, colaborando para consolidação do primeiro como padrão ouro no tratamento do câncer de canal anal. Embora nenhum deles tenha evidenciado algum grau de vantagem na sobrevida global com o tratamento combinado, todos mostraram melhores índices de controle local e sobrevida livre de colostomia.
Comparando nossa série com a literatura, observamos um número expressivo de pacientes diagnosticados com câncer do canal anal e tratados com radioquimioterapia em nossa instituição. $\mathrm{O}$ tempo médio de seguimento dos pacientes (51 meses), acima da média geral encontrada em estudos semelhantes reforça a importância desta revisão. Com relação ao tamanho do tumor, é comum no nosso serviço, recebermos pacientes com doença avançada. Cerca de $70 \%$ dos casos foram classificados como T3 ou T4 na época do diagnóstico. No entanto, mesmo tratando doença mais avançada, conseguimos, com radioquimioterapia concomitante, resultados favoráveis semelhantes aos encontrados na literatura mundial.

Levando-se em consideração que o fator que mais influenciou a resposta inicial e final ao tratamento foi o estadiamento do tumor, torna-se óbvia a necessidade de um melhor acesso da população ao atendimento médico especializado a fim de flagrar lesões em fases menos avançadas e, assim, aumentar as possibilidades de cura.

Com relação à toxicidade da radio e quimioterapia, pudemos observar que mais de $50 \%$ dos pacientes apresentou algum grau de intolerância ao tratamento, principalmente epitelite e diarréia que, em alguns casos, culminou com a interrupção temporária do esquema. No entanto, a morbidade apresentada não afetou o resultado final do tratamento na maior parte dos casos. Uma maior toxicidade é referida por publicações de instituições que utilizam a Mitomicina $\mathrm{C}$ no lugar do Cisplatino, escolha de nosso serviço, não apenas pela menor toxicidade como também por ser uma droga mais acessível financeiramente.

Para os pacientes que apresentam persistência da doença ou recidiva local após a radio e quimioterapia combinados, a ressecção anorretal é a modalidade de escolha do tratamento, podendo-se obter, segundo alguns estudos, cura em $50 \%$ dos casos. Alternativamente, utiliza-se um tratamento quimioterápico (5-FU e cisplatina) e radioterápico de auxílio (boost) com sucesso, evitando-se uma colostomia definitiva. Observamos em nossa casuística, resposta positiva ao boost em cerca de $33 \%$ dos pacientes submetidos a este tipo de tratamento após persistência da doença.

\section{CONCLUSÕES}

Esta revisão feita no Hospital Barão de Lucena corrobora com os resultados obtidos da literatura, con- 
Rev bras Coloproct Abril/Junho, 2010
Tratamento com Radio e Ouimioterapia do Carcinoma Epidermóide do Canal Anal: Experiência do Hospital Barão de Lucena Maurilio Toscano de Lucena e Cols.
Vol. 30 firmando a eficácia do tratamento radioquimioterápico exclusivos, com índice de resposta completo bastante elevado e morbidade aceitável. O principal ponto defendido é a preservação da função esfincteriana.
O tratamento cirúrgico ainda tem seu valor nos casos de persistência da lesão e/ou de recidiva local, com resultados satisfatórios.

ABSTRACT: Objectives: To present the results and analyze the variables involved in the treatment and prognosis of squamous cell carcinoma of the anal canal treated by radiotherapy and chemotherapy at the Hospital Barao de Lucena-SUS-PE. Methodology: Analysis of medical records of patients diagnosed with anal cancer treated by chemoradiation. The monitoring period was from June 1989 to June 2005. We included patients with histologically confirmed cancer of the anal canal, framed in stages I, II, IIIa and IIIb, underwent two cycles of chemotherapy with 5-fluorouracil (5-FU) at a dose of $1 \mathrm{~g} / \mathrm{m}^{2} /$ day continuous infusion 96 hours and cisplatin at a dose of $100 \mathrm{mg} / \mathrm{m}^{2}$ administered at 6 hours the second day of infusion of each cycle, administered on the first and third weeks of radiotherapy treatment regimen. Results: We evaluated records of 108 patients who met the criteria of the protocol. The mean follow-up was 51 months (1-182 months). There were more females $(81.5 \%$ of patients). The age ranged from 33 to 83 years (mean 59 years). The most common histological type was squamous cell carcinoma (80.6\% of cases). In 21 patients, was diagnosed Basaloid carcinoma. Regarding the degree of differentiation, the most prevalent type was moderately differentiated (61\% of patients with squamous cell carcinoma). The rate of initial complete response was $89.8 \%$. Eleven patients had persistent tumor after radiotherapy and chemotherapy. The initial response rate was lower in complete stages IIIa and IIIb compared to stages I and II with statistical significance $(\mathrm{p}<\mathbf{0 . 0 5})$. 14 patients developed recurrence, eight with local recurrence $(\mathbf{7 . 4 \%})$ and six (5.5\%) with lymphatic recurrence and distance. CONCLUSIONS: The chemoradiation treatment of unique cell carcinoma of the anal canal, have complete response rate very high with acceptable morbidity. Surgical treatment still has its value in cases of persistent injury and / or local recurrence, with satisfactory results.

Key words: Neoplasm of the anus, squamous cell cancer, radiotherapy, chemotherapy, combined chemotherapy.

\section{REFERÊNCIAS}

1. Nakamura, AR; Ferrigno, R; Salvajoli, JV; Nishimoto, IN; Davi Filho, WJ; Lopes, A. Tratamento conservador do carcinoma do canal anal. Revista do Colégio Brasileiro de Cirurgiões. Jan./Fev. 2005; 32 (1): 23-31.

2. Nadal, SR; Manzione, CR. Os agentes sexualmente transmissíveis e o câncer anorretal. Rev bras Coloproct, 2004; 24(3):274-77.

3. American Joint Committee on Cancer. Anal Cancer. In: Greene, FL; Page, DL; Fleming, ID; et al. (eds) - AJCC cancer staging manual. Springer-Verlag. 2001; 139.

4. Guimaraes, AP, Matos, D; Segreto, R, et al. Squamous cell carcinoma of the canal anal: analysis of 11 patients. Arq. Gastroenterol., Jan./Mar. 2001; 38(1):09-13. ISSN 0004-2803.

5. Myerson, RJ; Kong, F; Birnbaum, EH; Fleshman, JW; Kodner, IJ; Picus, J; Ratkin, GA; Read, TE; Walz, BJ. Radiation therapy for epidermoid carcinoma of the anal canal, clinical and treatment factors associated with outcome. Radiotherapy and Oncology. 2001; 61: 15-22.

6. Vuong, T; Devic, S; Belliveau, P; Muanza, T; Hegyi, G. Contribuition of conformal therapy in the treatment of anal canal carcinoma with combined chemotherapy and radiotherapy: results of a phase II study. Int J Radiation Oncology Biol Phys. 2003; 56(3):823-31.
7. Gerard, JP; Ayzac, L; Hun, D; Romestaing, P; Coquard, R; Ardiet, JM; Mornex, F. Treatment of anal canal carcinoma with high dose radiation therapy and concomitant fluorouracilcisplatinum. Long-term results in 95 patients. Radiotherapy and Oncology. 1998;46: 249-56.

8. Fuchshuber, PR; Rodriguez-Bigas, M; Weber, T; Petrelli, NJ. Anal canal and perianal epidermoid cancers. J Am Coll Surg. 1997; 185 (5): 512-24.

9. Ryan, DP; Compton, CC; Mayer, RJ. Carcinoma of the anal canal. N Engl J Med 2000; 342(11): 792-8.

10. Epidermoid anal cancer: results from the UKCCCR randomized trial of radiotherapy alone versus radiotherapy, 5-fluorouracil, and mitomycin. UKCCCR anal Cancer Trial Working Party. Lancet, 1996; 348(9034): 1049-54.

11. Flam, MS; John, MJ; Peters, T; et al. Radiation and 5fluorouracil (5FU) vs. radiation, $5 \mathrm{FU}$, mitomycin - C (MMC) in the treatment of anal canal carcinoma: preliminary results of a phase III randomized RTOG/ ECOG intergroup trial. Am J Clin Oncol, 1993; 12: 192.

12. Frisch, M; Glimelius,B; Brule, AJCVD; Wohlfahrt, J; Meijer, CJLM; et al. Sexually Transmitted infetion as a cause of anal cancer. N Engl J Med. 1997; 337:1350-8.

13. Youk, EG; Ku, JL; Park, JG. Detection and typing of human papillomavirus in anal epidermoid carcinomas: sequence 
variation in the E7 gene of human papillomavirus type 16. Dis Colon Rectum, 2001; 44 (2): 236-42.

14. Akbari, RP; Paty, PB; Guillem, JG; Weiser, MR; et al. Oncologic outcomes of Salvage Surgery for epidermoid carcinoma of the anus initially managed with combined modality therapy. Dis Colon Rectum, 2004; 47: 1136-44.

15. Nilsson, CS; Goldman,S; Glimelius,B. Salvage abdominoperineal resection in anal epidermoid cancer. Br J Surg, 2002; 89: 1425-29.
Endereço para correspondência: MAURILIO TOSCANO DE LUCENA

Rua Frei Jaboatão, 180/1201. Torre. Recife-PE. CEP: $50710-030$

Fone: (81) 3445-4932 / (81) 9174-9541

E-mail:mtlucena@oi.com.br 